\title{
Drieu La Rochelle ou le MAL(E) ETRE
}

\author{
François-Xavier EYGUN \\ Mount Saint Vincent University, Halifax
}

Un certain nombre d'hommes et d'écrivains français de la première moitié du XXème siècle, dont Pierre Drieu la Rochelle particulièrement, ont été qualifiés de phallocrates sereins. Ceux-ci, sûrs de leurs bons droits dans une société patriarcale, s'interrogèrent pourtant beaucoup sur la femme, comme moyen peut-être, de répondre aux questionnements liés à leur propre masculinité. Elizabeth Badinter dans son essai $X Y$, pourrait résumer par la citation suivante: "Le premier devoir pour un homme est: ne pas être femme" (Badinter 66), l'incessant questionnement sur la femme et sur soimême que Pierre Drieu la Rochelle a entrepris à travers, principalement, toute son oeuvre romanesque. Ce questionnement de l'autre sexe comme moyen de définir le sien propre, est intéressant à plus d'un point, car il remet en question ce qui aurait pu être la plus importante mise en scène (ou supercherie) du patriarcat, à savoir que la masculinité ne se questionne pas et que c'est à partir de cette assurance masculine que se définit la féminité, alors qu'il s'agit plutot du contraire, pour Drieu la Rochelle en tous les cas. Ce que d'ailleurs Badinter confirme et precise: "Contrairement à l'idéologie du patriarcat, ce ne sont pas les hommes qui sont les premiers référents de l'humanité, mais les femmes. C'est par rapport à elles et contre elles qu'ils se définissent" (Badinter 23). La misogynie (ou définie comme telle) de Drieu la Rochelle peut, sans doute, mieux se comprendre et s'interpréter, si l'on fait de cette citation le point de départ de la quête identitaire contenue dans l'oeuvre romanesque de cet homme couvert de femmes (titre de l'un de ses romans, publié en 1925).

Presque cinquante ans après son suicide (1945), Pierre Drieu la Rochelle n'en a pas fini de hanter la scène littéraire et politique (ou la combinaison des deux): toute- 
fois son oeuvre romanesque dépasse le personnage de l'auteur et c'est son monde imaginaire mais aussi profondément autobiographique que nous solliciterons. Il faut savoir en effet, qu'auteur et narrateur ne font qu'un dans presque toute l'oeuvre de Drieu, et que par conséquent (et comme l'a montré Dominique Desanti entre autre dans son livre: Drieu la Rochelle ou le séducteur mystifié), on peut retrouver les péripéties du narrateur comme un calque de celles de l'auteur dans la plupart des romans de Drieu. Autrement dit, la biographie et le monde romanesque se rejoignent et s'éclairent l'un l'autre.

Les rapports homme/femme et par là l'interrogation sur l'être féminin et masculin, paraissent être un thème central de l'oeuvre rochellienne. Comment être homme, comment être femme? Ces questions parcourent tous les textes et si parfois quelques éléments de réponse peuvent être proposés, ceux-ci débouchent aussi sur plus de questions, et sur finalement ce qui demeure encore et toujours à définir.

A travers l'essai d'Elizabeth Badinter $X Y$, il sera tenté une relecture de l'oeuvre de Drieu, à la lumière de ce que l'on reconnaît aujourd'hui comme "le mâle-être" et qui fut longtemps (sinon depuis toujours) soit nié, soit l'apanage de la problématique féminine. Ainsi il sera plus aisé de saisir comment le questionnement sur la femme, chez Drieu la Rochelle, est aussi, et peut-être surtout, une interrogation sur l'homme à la lumière de ce qui a été précédemment cité, à savoir que le principal devoir pour un homme est de ne pas être une femme, et que pour paraphraser, et Elisabeth Badinter, et Simone de Beauvoir, on ne naît pas homme, on le devient. La grille critique dévelopée par E. Badinter a cela de remarquable, c'est qu'elle s'applique comme un gant à cette quête de l'identité masculine qui se lit dans l'oeuvre de Drieu la Rochelle. Par conséquent, elle sera adoptée comme telle, pour pouvoir démontrer l'inquiétante absence d'assurance masculine dans l'oeuvre de Drieu et les conséquences que cela a pu avoir pour un homme et un écrivain français à une époque donnée.

Né en 1893 et mort en 1945, Drieu la Rochelle a commencé par écrire et publier deux recueils de poèmes (Interrogation, 1917, et Fond de cantine, 1920) avant de se lancer dans l'aventure romanesque en 1921 avec Etat civil. Ses premiers poèmes, comme l'indique le titre du recueil de 1917, tentent de comprendre et d'expliquer la naissance de l'homme adulte au contact de la guerre, et ce que signifie celle-ci dans le développement de la masculinité. Par conséquent ces premiers écrits sont une forme de témoignage du devenir de l'homme dans sa particularité masculine, et le fait que ce soit à travers la guerre que cette quête de l'identité se précise, n'est pas sans rap- 
procher ces poèmes à une étape décrite par Badinter comme celle de l'épreuve (la troisième des trois étapes), qui sert à détacher le jeune mâle du monde féminin de l'enfance et à lui prouver qu'il est devenu homme.

Selon Badinter, la recherche de l'identité masculine met l'accent sur la différenciation: "Je ne suis pas ma mère, je ne suis pas un bébé, je ne suis pas une fille" (Badinter 77), alors que le processus d'identification féminine serait relationnel. Pour la fille, par exemple, cette relation à la mère, "[...] est à la base d'une identification avec son propre sexe, alors que pour le garçon elle est une inversion des rôles ultérieurs" (Badinter 77). Cela a pour conséquence que la masculinité est une réaction, une protestation, tout d'abord contre la passivité et l'impuissance du nouveau-né et de l'enfant (la protoféminité du bébé humain) qui doit se détacher du désir passif d'être materné, et ensuite, et aussi, comme une forme de différenciation à l'égard du monde féminin maternel:"Pour devenir un homme, il devra apprendre à se différencier de sa mère et à refouler au plus profond de lui cette passivité délicieuse où il ne faisait qu'un avec elle" (Badinter 63). Ce passage (ou différenciation/transmutation), prend la forme dans de nombreuses sociétés, de rites d'initiation, et il s'effectue en trois étapes que Badinter résume ainsi:

Celle-ci comporte trois étapes plus douloureuses les unes que les autres: la première est la séparation d'avec la mère et le monde féminin; la seconde consiste du transfert dans un monde inconnu; et finalement la troisième étape achève le processus d'identification masculine grâce au passage d'épreuves dramatiques et publiques (Badinter 94).

L'enfance de Drieu la Rochelle, telle que romancée dans Etat civil (1922) et Rêveuse bourgeoisie (1937), est une enfance privée de la présence d'un père volage et irresponsable où les repères de masculinité sont flous et plutôt (mal) représentés par un grand-père qui prend en charge financièrement la famille de Drieu. Quant à sa mère, elle semble être vouée à la souffrance et à la passivité, lesquelles sont dues, entre autres, aux carences du mari, et ensuite au rôle protecteur des grands-parents, qui perpétuent un peu plus cette passivité en prenant en charge les soucis d'argent et de stabilité affective.

Cette absence de modèle masculin et le déséquilibre du rôle féminin qui en est la contrepartie, correspond dans $X Y$ a un malaise de la société qui ne date pas d'aujour- 
d'hui, puisque Badinter en fait remonter l'origine au début du XXème siècle corroborant ainsi le témoignage de Drieu sur sa propre famille. Cette absence du père, selon Badinter, prive le petit garçon de son initiateur mâle et a pour conséquence une recherche identitaire qui oscille entre le modèle féminin pourvoyeur d'une sécurité et aussi d'une passivité - affective, et cette quête du père absent: le résultat ne peut être qu'ambigu et source de conflit entre une recherche de soi par rapport à un genre masculin ou féminin, et le rôle que la société impose à ces mêmes genres masculin et féminin. A telle enseigne que le narrateur de Rêveuse bourgeoisie, devient en fait une narratrice en cours de roman, et que quand Drieu la Rochelle, à l'âge de 42 ans, évoque son enfance, il la conjugue au féminin.

C'est d'ailleurs une des constantes de l'univers romanesque rochellien, cette ambivalence identitaire, qui passe d'un extrême à l'autre. Soit le narrateur se gausse d'une masculinité presque épique et mythique (comme dans La Comédie de Charleroi) ou alors se glisse dans la peau d'une femme futile comme la narratrice de Rêveuse bourgeoisie. Ces deux romans sont d'ailleurs des étapes importantes dans le cheminement de la quête identitaire, l'un (Rêveuse bourgeoisie) étant une tentative d'exorciser et de comprendre le passé, l'autre (La Comédie de Charleroi) de témoigner de cette initiation à la masculinité que fut la guerre.

Badinter souligne aussi que le devenir masculin se fait par réaction, par révolte contre le modèle féminin de l'enfance, et que cette révolte doit s'accomplire en temps utile:

Aux alentours de la pré-adolescence, le garçon a le devoir de sortir de l'enfance indifférenciée. Au regard de la plupart des sociétés, devenir un homme adulte est problématique. Par opposition à la femme qui est, l'homme, lui, doit être fait... Autrement dit, le devenir homme est une fabrication volontariste, et on peut se demander [...] si la masculinité des fils s'éveillerait jamais si elle n'était pas forcée à un moment déterminé de son développement (Badinter 92).

L'adolescence de Drieu, du moins ce que nous en savons à travers ses écrits, se passa à l'ombre de ses grands-parents et au milieu de tiraillements familiaux dus au peu d'entente entre ses parents et aux problèmes financiers de sa famille. Cette adolescence qui aurait dû être un apprentissage de la masculinité, sera, contrairement à ce que souligne la citation précédente, une dilution un peu plus consciente du genre ma- 
sculin: sorte de signifiant sans signifié comme le mentionne Badinter en s'interrogeant sur la condition d'homme.

Dominique Desanti, dans son livre sur Drieu la Rochelle, résume ce que fut l'enfance de celui-ci, telle qu'elle a été décrite dans les deux romans autobiographiques, Etat civil et Rêveuse bourgeoisie:

Drieu a créé [sous l'influence de Lacan vers 1930] son double féminin. Il lui confère ce qu'il sait à présent en lui ; le goût d'être au centre du spectacle, la tentation de l'argent donné par le partenaire érotique, la nostalgie de l'amour absolu chaque fois rejeté. Face aux grands-parents, aux parents, à lui-même, nourris de son vécu, il dresse, il incarne son fantasme: être femme; être l'Autre. Réduire la femme, partenaire, obsession, assujettissement, en s'identifiant à elle (Desanti 60).

Avant même d'avoir compris cette double postulation de son être, Drieu adolescent, comme beaucoup d'autres adolescents, songe déjà au suicide, et tentera finalement de se sortir de cette absence de signifié en s'engageant dans l'armée en 1913. Ce sera aussi une façon d'oublier ses échecs, qu'ils soient universitaires (il échoue à l'examen de Sciences Politiques) ou sexuel (première tentative avec une prostituée à la veille de ses dix-huit ans) qui le laissera, le reste de sa vie, étrangement attiré par ce genre de commerce, comme s'il était incapable de remplir sa fonction d'homme dans une relation autre que tarifée.

Par rapport aux trois étapes d'identification: la séparation d'avec la mère et le monde féminin, le transfert dans un monde inconnu, et le passage par des épreuves dramatiques et publiques, on ne peut que constater que les deux premières ne furent guère réussies. Par contre, la troisième étape, celle de l'initiation fut, en ce qui concerne Drieu la Rochelle et bien des hommes des sociétés occidentales de cette époquelà, particulièrement dramatique et riche de conséquences douloureuses, pour ces jeunes hommes, et pour la société en général.

Cette troisième étape ou rite d'initiation commence pour cette génération par le service militaire et puis, en ce qui concerne Drieu la Rochelle, tout de suite la guerre. Comme si, en dehors d'autres considérations politiques, la guerre était pour à peu près chaque génération, un moyen d'initier les jeunes mâles à leur état d'homme. 
Drieu la Rochelle fut engagé volontaire en 1913, et ne sortit de l'armée qu'en 1919 , trois fois blessé. Déjà pénétré de relents nietzschéens avant la guerre, du nécessaire rapport de force entre les êtres (le rapport oppositionel), du besoin de se trouver un ennemi, il découvrira dans la guerre ce qui marquera une bonne partie de sa vie et de son oeuvre: c'est-à-dire l'histoire de son rapport avec les femmes et la société en général qui représente pour lui l'apothéose des valeurs féminines.

L'armée lui ouvre les portes du monde masculin et la première guerre mondiale qui débutera peu après, une révélation de sa nature, ou du moins de facettes de sa nature, car le problème identitaire ne sera jamais résolu. Cette guerre lui permettra de découvrir le rôle traditionnel du guerrier et lui donnera l'occasion d'être un homme, un vrai! Il en arrivera à faire de cet état l'essence de sa quête identitaire:

Quand la société s'éloigne de la guerre, toute passion et singulièrement tout amour meurt bientôt. Qu'est-ce qu'un amant qui ne peut plus tuer son rival et que son rival ne peut pas tuer? Qu'est ce qu'un homme qui n'est pas plus fort que la femme, qui n'est pas promis à des épreuves plus fortes? Comment une femme peut-elle supporter encore l'enfantement si son mari ne supporte pas le combat? L'homme n'étant plus occupé par la guerre fait trop l'amour, il se fatigue, il devient passif. Inverti avec la femme, il peut aussi bien l'être avec l'homme. Et la femme caressant l'homme peut aussi bien caresser la femme.

Si l'homme ne risque pas sa vie dans le combat, il ne risquera bientôt plus sa vie dans la paternité. Car faire un enfant, c'est à demi mourir, c'est à demi s'effacer, c'est mutiler à jamais son égoïsme. [...] A partir du moment où l'homme ne risque plus la mort, il ne peut plus croire dans les dieux, car ils représentent le sentiment de la vie affrontant la mort et la surmontant... (Drieu la Rochelle, Sur les Ecrivains 224).

Cette longue citation tirée de son essai Sur les écrivains (publié en 1964), fait du combat l'occupation essentielle et nécessaire de l'homme, celle qui justifie et sa vie et son rôle. Mais le moins que l'on puisse dire, en ce qui concerne Drieu la Rochelle, c'est que cette projection de soi dans le combat, sorte de flirt avec la mort, qu'elle provienne du contact avec le monde féminin ou du combat guerrier, n'est que la réponse à un dilemme ontologique qui oscille entre deux pôles: soit la mort par la femme et la paternité, ou soit un long ennui, qui est, pour reprendre un mot de la cita- 
tion: dépérissant. Entre ces deux pôles, il y a le combat, combat pour retarder la mort (ou la passivité), pour tenter vainement de s'en jouer, pour caresser l'illusion que cette mort peut être contrôlée, dominée par l'activité combattante. Drieu, dans cette citation, s'arrête aussi sur la passivité, qui est pour lui synonyme d'inversion, de réduction des genres masculin et féminin à un rôle flou qui tend à l'homosexualité, et que Drieu considère comme une décadence de la société, mais aussi une disparition ontologique de l'altérité qui crée la vie par la fusion de ses contraires.

Cette méfiance, pour le moins, envers la passivité, correspond à cet état d'opposition qui selon Badinter, différencie la recherche de la masculinité. L'homme en devenir doit se prouver en s'opposant, tout d'abord à sa mère, ensuite en se distinguant de façon radicale d'avec le sexe féminin et finalement, il doit "[...] se prouver qu'il n'est pas homosexuel, donc qu'il ne souhaite pas désirer d'autres hommes ni en être désiré. Dans notre civilisation prédomine l'idée qu'on est vraiment homme si on préfère une femme. Comme si posséder une femme renforçait l'altérité désirée en éloignant le spectre de l'identité: avoir une femme pour ne pas être une femme" (Badinter 125-126).

Ce que souligne Badinter dans ce besoin d'opposition et de désir d'avoir l'autre pour ne pas être l'autre, conduit, chez des êtres comme Drieu conscients de leur ambivalence masculine/féminine, à un refoulement soit de l'une ou de l'autre composante de cette altérité. Il ne s'agit pas chez Drieu, du moins dans son oeuvre, du déni d'une bisexualité désirée, mais plutôt d'une difficulté à se conformer au rôle masculin adulte. Toutefois, le résultat est un homme décomposé, fragmenté, ou, comme le nomme Badinter: l'homme coupé en deux. Il s'ensuit dans son analyse de l'homme coupé en deux, une association politique qui concerne très particulièrement Drieu la Rochelle:

Le cas limite de l'homme coupé en deux est celui du mâle fasciste hitlérien décrit par Klaus Theweleit: "Les hommes étaient alors déchirés entre un intérieur (femelle) et un extérieur (mâle), des ennemis mortels... Ce que le fascisme promettait aux hommes était la réintégration de leurs composantes hostiles de manière tolérable, à savoir par la domination de l'élément 'femelle hostile"' (Badinter 156).

Que le "mâle-être" puisse conduire au fascisme s'applique à Drieu, qui se sera fourvoyé à partir des années trente dans ce mouvement, d'une façon certes ambiguë 
puisque son engagement sera composé de revirements et d'autres aspects comme la vision d'une Europe unie qui est en soi prémonitoire, mais il n'en reste pas moins que cette composition, cette fabrication d'une masculinité à travers le fascisme a marqué l'Europe de la première moitié du XXème siècle. Ce fut peut-être la réponse à une crise identitaire où finalement le culte de la force serait resté la plus constante compensation des affaiblis par cette épidémie de "mâle-être." A cela s'ajoute une analyse plus psychanalytique développée par Badinter selon la théorie freudienne, et qui propose de voir dans le fascisme et ses corrolaires, un complexe de castration qui assimile misogynie et antisémitisme: "L'analogie entre la femme et le juif, la coïncidence entre la misogynie et l'antisémitisme (auxquels il faudrait ajouter l'homophobie) se retrouvent chez nombre d'écrivains du XXème siècle. Des contemporains de Weininger à Henry Miller en passant par D.H. Lawrence, E. Hemingway et Drieu la Rochelle, on constate que l'un va rarement sans l'autre" (Badinter 157).

Sans vouloir enfermer Drieu la Rochelle dans un modèle d'analyse, donc réducteur, qui a ses limites quand on évoque un créateur littéraire, on peut toutefois constater que le devenir masculin, à travers et l'oeuvre romanesque de Drieu, et à la lecture de sa biographie, témoigne que le processus identitaire ne s'acquiert pas sans conséquences sur la société. De plus, sans tenter outre mesure de victimiser Drieu dans sa quête identitaire, force est de montrer du doigt la société qui a engendré de tels poisons. Drieu d'ailleurs dans ses écrits, tentera de fournir un antidote par la critique constante et acerbe d'une société qu'il juge décadente (le roman Gilles, par exemple, publié en 1939). Il ira même plus loin, puisque dès 1943, il aurait pu, comme beaucoup d'autres changer de camp, et hurler avec les loups, mais il préférera plutôt, par ennui ou par conviction - qui pourrait dire leur lien étroit? - il préférera donc la mort dans ce qu'il avait depuis longtemps analysé comme une voie sans issue.

L'importance du "mâle-être" chez Drieu la Rochelle peut paraître déterminant pour illustrer une époque et une société particulière, grâce à une oeuvre littéraire. Maintenant, par juxtaposition, l'on peut se demander ce qu'est la nôtre, ce que sera la nôtre, qui a fait d'un Rambo sa sublimation fantasmatique du modèle de la virilité? 


\section{Bibliographie}

Badinter, Elisabeth. XY. De l'identité masculine. Paris: Odile Jacob, 1992.

Desanti, Dominique. Drieu la Rochelle ou le séducteur mystifié. Paris: Flammarion, 1978.

Drieu la Rochelle, Pierre. Sur les Ecrivains. Paris: Gallimard, 1964.

. L'Homme couvert de femmes. Paris: Gallimard, 1925.

Gilles. Paris: Gallimard, 1965.

Rêveuse bourgeoisie. Paris: Gallimard, 1937.

. La Comédie de Charleroi. Paris: Gallimard, 1934.

. Blêche. Paris: Gallimard, 1928.

Interrogation. Paris: Gallimard, 1917.

. Fond de cantine. Paris: Gallimard, 1920.

. Etat civil. Paris: Gallimard, 1921. 